\title{
Das Problem der Systematisierung der anorganischen Verbindungen.
}

\author{
Von \\ JAMES LOOKE. ${ }^{1}$ \\ Mit 4 Figuren im Text.
}

Es würde wohl schwierig sein, heute noch einen in der anorganischen Chemie bewanderten Chemiker zu finden, der nach einem sorgfältigen Studium des periodischen Systemes die Ansicht verträte, dafs dasselbe nach allen Richtungen befriedigte. Die Mängel des Systemes lassen sich in zwei Gruppen einteilen. Die erste Gruppe umfalst solche Thatsachen, die in direktem Widerspruch zum System stehen, wie etwa das Atomgewicht des Tellurs, das Verhalten des Argons u. s. w. Die zweite Gruppe setzt sich zusammen aus Analogien solcher Verbindungen, welche nach der Stellung der entsprechenden Elemente im System keinerlei Analogie zeigen sollten. In der Litteratur finden sich meist nur die zur ersten Gruppe gehörigen Widersprüche diskutiert; die zweite - bei weitem grölsere - Klasse findet kaum Erwähnung, obgleich die Thatsachen, die sie umfafst, sich auf fast alle die Elemente beziehen, die in mehr als einer Oxydationsstufe auftreten. Es ist bisher sogar kaum ein Versuch gemacht worden, die Punkte dieser Art, in denen dits System Schwächen zeigt, zu sichten und zu systematisieren.

Nur ein bemerkenswerter - wenn auch indirekter - Versuch nach dieser Richtung scheint vorzuliegen und zwar in der von ABEgG und BODLÄNDER ${ }^{2}$ aufgestellten Theorie; nach dieser sollen die verschiedenen Eigenschaften der anorganischen Salze zu betrachten sein als Funktionen der Elektroaffinität der in ihrer Lösung ent-

1 Ins Deutsche übertragen von J. KoppeL.

${ }^{2} Z$. anorg. Ohem. 20, 453. 
haltenen Ionen, d. h. als Funktionen der Intensität, mit welcher die verschiedenen Ionen ihre positiven oder negativen Elektrizitätsladungen festhalten. Wenn diese Theorie richtig wäre, so könnte man ruhig sagen, dafs die anorganische Chemie jetzt in eine neue Epoche einträte. Leider aber ist sie nicht haltbar. Die von den Verfassern angeführten Beispiele sind in fast allen Fällen willkürlich, und oft zeigen sie bei der Prüfung auf andere Eigenschaften als die gewählten einen direkten Widerspruch gegen die Schlüsse der Verfasser. ${ }^{1}$ Wenn aber auch die theoretischen Deduktionen der erwähnten Arbeit als fehlerhaft betrachtet werden müssen, so sind wir doch den Verfassern zu unendlichem Dank verptlichtet; denn - absichtlich oder unabsichtlich - fördern sie auf jeder Seite eine solche Flut von Fragen zu Tage, dals wir durch unsere Unfähigkeit zur Beantwortung derselben immer wieder darauf hingewiesen werden, wie unzulänglich das periodische System in seiner vorhandenen Gestalt ist als vollständige Basis zur Systematisierung der anorganischen Verbindungen. Diese Fragen werden mit der Zeit gelöst werden, sie können jedoch nur au*gearbeitet werden auf Grund quantitativer Daten.

ABEGG und BoDläNDER's Theorie enthält drei wichtige Punkte: nämlich, dafs erstens die Löslichkeit der Salze mit steigender Elektroaffinität der Ionen zunimmt; dals zweitens gleichzeitig die Tendenz zur Bildung komplexer Kationen abnimmt; und dals schlielslich drittens auch die Tendenz zur Bildung komplexer Anionen abnimmt.

Dies sind drei Eigenschaften von sehr grofser Wichtigkeit. Wenn wir beispielsweise ganz allgemein die Reaktionen des Silberions beschreiben wollen, so dürfen wir nicht solche Charakteristika, wie die Eällung als Silberchlorid auswählen, welche auf den Eigenschaften eines Salzes basieren, sondern wir müssen solche Reaktionen nehmen, die alle Silbersalze zeigen, und deren Ausnabmen auf Grund der Gesetze der Massenwirkung und des Löslichkeitsproduktes erklärt werden können. Solch ein allgemeines Charakteristikum ist die Bildung von Metallaminen. Nach allen vorbandenen Daten ist die Existenz des komplexen Silberkations - etwa $\left[\mathrm{Ag}\left(\mathrm{NH}_{3}\right)_{2}\right]^{\circ}$ - unabhängig von dem sauren Radikal des Salzes, und jede Ausnahme von der Regel, dafs Silbersalze in Ammoniak löslich sind, ist nicht auf eine spezifische Eigenschaft des Salzes zurückzuführen, sondern

'Am. Chem. Journ. 27, 105. 
lediglich seinem äulserst kleinen Löslichkeitsprodukt zuzuschreiben. Wiederum finden wir nur sehr wenig Ausnahmen für die allgemeine Regel, dafs Silbersalze praktisch in Wasser unlöslich sind. In DAMMER's Handbuch sind nur fünf Säuren angegeben, deren normale Silbersalze sich reichlich in der Kälte lösen; und da jene Eigenschaft allgemeiner Natur ist, so muls sie ihre Ursache in den Eigenschaften des einwertigen Silbers selbst haben, und nur wenig von der Natur der Anionen beeinflufst werden. Weiterhin finden wir, dafs fast alle Silbersalze, die selbst in Wasser unlöslich sind, sich in Lösungen von Alkalisalzen des gleichen Anions lösen. Die Eigenschaft, komplexe Anionen zu bilden (wie z. B. in dem Salz $\mathrm{K}, \mathrm{Ag}(\mathrm{CN})_{2}{ }^{\prime}$ ), ist allgemein, wie die der geringen Löslichkeit und der Bildung komplexer Kationen; und diese drei Eigenschaften, zusammen mit der Unbeständigkeit beim Erhitzen, beschreiben qualitativ die Reaktionen fast jeden Silbersalzes.

Wir hätten also in der Elektroaffinität ein umfassendes Prinzip, auf welches die wichtigsten Eigenschaften einer Verbindung bezogen werden, ohne Rücksicht auf die Natur des Elementes und auf seine Valenz in der fraglichen Verbindung. Ist nun solch ein Prinzip sei es die Elektroaffinität oder das Atomgewicht, oder eine andere noch unbekannte Eigenschaft - richtig und durchaus anwendbar? Wenn das der Fall ist, so mülsten die Verbindungen des Elementes eine bestimmte Beziehung zwischen den oben diskutierten drei Eigenschaften zeigen. Je löslicher die Verbindung ist, um so weniger sollte sie zur Bildung komplexer Salze neigen; je leichter sie Ammoniakadditionsprodukte bildet, um so leichter sollte sie auch Doppelsalze bilden und um so geringer sollte die Löslichkeit ihrer Salze sein.

Um nun diese Frage näher zu prüfen, wollen wir die Reaktionen einiger Metalle von gleichen Valenz betrachten. Zuerst formulieren wir so kurz wie möglich die Eigenschaften der Natriumsalze. Mit sehr wenigen Ausnahmen sind sie leicht in Wasser löslich. Praktisch treten sie weder in komplexe Anionen noch in komplexe Kationen ein. Beim Erhitzen sind sie entweder beständig, oder sie gehen in andere Salze über, nie aber in das Oxyd. Natriumchloid z. B. ist löslich in Wasser; da es jedoch weder komplexe Kationen noch Anionen bildet, so wird es aus seiner konzentrierten Lösung durch Chlorwasserstoffsäure gefällt. Die Kaliumsalze lösen sich gleichfalls reichlich in Wasser, und indem sie diese eine Eigenschaft mit den Natriumsalzen gemeinsam haben, zeigen 
sie auch alle anderen oben aufgezähIten Eigenschaften. Das Gleiche gilt für Rubidium und Cäsium. Es müssen deswegen also diese Eigenschaften wirklich an einander direkt ver$\mathrm{knüpft}$ sein; und zwar müssen sie abhängig sein von einer allen Alkalimetallen inhärenten Ursache. Diese Eigenschaften weichen ab von den Eigentümlichkeiten, die das Verhalten der Silbersalze charakterisieren, nämlich: Unlöslichkeit, Bildung komplexer Kationen und Anionen, Unbeständigkeit beim Erhitzen. Sie sind ihnen diametral entgegengesetzt. Cuprochlorid wiederum ist in Wasser unlöslich, dagegen löslich in Ammoniak und in Kaliumchlorid; endlich sieht man, dals, abgesehen von der Neigung, sich höher zu oxydieren, jedes andere bekannte Cuprosalz fast genau dieselben Eigenschaften hat, wie das entsprechende Silbersalz, dagegen aber keine von den Eigenschaften, die die Natriumverbindungen charakterisieren.

Wenn wir weiterhin irgend ein anderes einwertiges Metall prüfen, so finden wir, dafs seine Verbindungen, soweit sie existenzfähig sind, unter eine dieser beiden Gruppen fallen. Einwertiges Quecksilber und einwertiges Gold, beispielsweise, welche allerdings nur sehr unbeständige Verbindungen liefern, die grofse Neigung zeigen, in Verbindungen des zwei- bezw. dreiwertigen Metalles überzugehen, sind in ihren Eigenschaften mit den Silberverbindungen identisch, wenn man absieht von ihrer Fähigkeit, sich zu oxydieren. Die einzige Ausnahme ist Thallium. Dies Element bildet in Form von Thallosalzen wie die Natriumverbindungen fast keine komplexe Anionen oder Kationen - seine Salze sind aber, wie die Silbersalze, fast alle schwer löslich.

Es sollen nunmehr die zweiwertigen Metalle betrachtet werden, wobei wir uns auf die Verbindungen der besser bekannten Elemente beschränken. Kann man sie, wie die einwertigen Elemente, in zwei oder drei einfache Gruppen einteilen? Wenn dies der Fall ist, finden wir dann dieselben Eigenschaften in Beziehung zu einander wie dort? - Die letztere Frage ist von grofser Wichtigkeit; denn von ihr hängt die weitere Frage $a b$, ob wir berechtigt sind, das Prinzip der Elektroaffinität oder eine verwandte Eigenschaft als Basis für ihre Klassifikation zu benutzen, ohne Rücksicht auf ihre Valenz. Finden wir wieder die Bildung komplexer Anionen und komplexer Kationen mit geringer Löslichkeit verbunden, so können wir den Vailenzwert vernachlässigen. Ist dies aber nicht der Fall, sind vielmehr bei den zweiwertigen Metallen diese allgemeinen Eigenschaften in anderer Weise verbunden wie bei den einwertigen Ele- 
menten, dann mufs der erste Schritt zur Feststellung der Primärursache der Eigenschaften von anorganischen Körpern darin bestehen, sehr scharf die einwertigen Metalle von den anderen zu sondern. Mit anderen Worten, wir müssen ein Element in einem Valenzzustand als eine durchaus andere Primärform der Materie auffassen, als dasselbe Element in einer anderen Oxydationsstufe. lm ersten Falle würde unser Problem verhältnismälsig einfach sein; im zweiten wäre es komplizierter, und dies ist leider der Fall.

Zinksalze werden bekanntlich aus ihren Lösungen durch Ammoniak nicht gefällt und fast sämtliche schwer lösliche Zinkverbindungen lösen sich in Ammoniak. Zinkcyanid löst sich in Kaliumcyanid, wobei sich das komplexe Ion $\mathrm{Zn}(\mathrm{CN})_{4}$ bildet. Diese Reaktion ist nur ein bekanntes Beispiel für die Fähigkeit fast aller Zinkverbindungen zur Bildung von Doppelsalzen. Diese zwei allgemeinen Eigenschaften, Bildung von Doppelsalzen und von komplexen Kationen, sind nun charakteristisch für die Silbersalze, bei denen sie mit geringer Löslichkeit vereinigt sind. Bei den Zinksalzen dagegen sind sie mit grofser Löslichkeit verbunden. Betrachtet man die Reihe der Zinkverbindungen, so findet man, dafs sich die Salze fast jeder Säure mit Leichtigkeit lösen, z. B. das Nitrat, Chlorid, Fluorid, Sulfat, Chromat, Selenat, Thiosulfat, Hypophosphit, Jodat, Phosphit, Silikofluorid, Sulfit, Dithionat, Nitrit, Hyponitrit u. s. w. Die entsprechenden Silbersalze sind fast alle unlöslich. Wir wenden uns jetzt zu den Baryumverbindungen: DaMMER's Handbuch erwähnt nur ein Baryumdoppelsalz, die Verbindung $\mathrm{K}_{2} \mathrm{Ba}\left(\mathrm{NO}_{2}\right)_{4}$. Doppelverbindungen des Chlorids, Sulfats und Cyanids sind unbekannt; auch wird die Löslichkeit der Baryumsalze durch Gegenwart von Ammoniak nicht merklich beeinflufst. Demnach fehlt dem Baryum praktisch die Fähigkeit zur Bildung von Doppelsalzen und von komplexen Kationen. Das Gleiche gilt für das Natrium, dessen Salze gleichzeitig in Wasser löslich sind. Die Baryumsalze dagegen sind durchweg durch geringe Löslichkeit charakterisiert, eine Eigenschaft, die bei den einwertigen Metallen, wie wir sahen, mit der Fähigkeit zur Bildung komplexer Ionen verbunden war. Die Baryumsalze der sämtlichen oben aufgeführten Säuren sind weniger löslich als die entsprechenden Zinksalze; die Natriumsalze dagegen sind löslicher als die Silbersalze. - Daraus ergiebt sich, dals bei Elementen von verschiedener Valenz die drei erwähnten Eigenschaften nicht notwendig in der gleichen Weise miteinander verbunden sein müssen. 
Vergleicht man nun die Löslichkeiten der erwähnten Zinksalze einzeln mit denen der Kobaltsalze, so findet man, dafs sie in allen Fällen qualitativ gleich sind. In den meisten Fällen haben die Salze den gleichen Gehalt an Krystallwasser und sind isomorph. Mit Mangan zeigt sich die gleiche Übereinstimmung und ebenso mit Magnesium, zweiwertigem Eisen, zweiwertigem Kupfer, Nickel und Cadmium. Alle diese Metalle geben mit den Alkalisalzen Doppelsalze, die Reihe für Reihe mit denen des Zinks isomorph sind. Weiterhin ist bei allen diesen Metallen die Bildung komplexer Kationen in Lösung beobachtet worden, ausgenommen das Magnesium, welches aber wahrscheinlich in Gegenwart eines grofsen Ammoniaküberschusses zur Bildung von Metallaminen befähigt ist. Man ersieht, also, dafs das gleichzeitige Hervortreten von Leichtlöslichkeit, Bildung von komplexen Kationen, und Bildung von komplexen Anionen, im Falle der Zinksalze, nicht gerade zufällig ist, sondern dafs diese drei allgemeinen Eigenschaften in engster Verbindung mit einander stehen. Denn wenn die Salze irgend eines zweiwertigen Metalls die eine Eigenschaft besitzen, so scheinen sie auch die anderen besitzen zu müssen. Fehlt, aber, eine dieser allgemeinen Eigenschaften, so bleiben alle derselben aus. Indem Calciumsalze keine beständige Amine bilden, weichen sie von den Zinksalzen durchaus ab, um sich mit den Baryumverbindungen in der entgegengesetzten Gruppe einzureihen.

Für den Zusammenhang bestimmter Eigenschaften bei den Verbindungen von Elementen in noch höheren Oxydationsstufen, sei folgendes Beispiel angeführt. Im Jahre 1867 beobachtete Roscow, dafs das Vanadintrichlorid $\mathrm{VCl}_{3}$ sich in Wasser mit grüner Farbe löst. Das war alles, was wir $20 \mathrm{Jahre} \mathrm{lang} \mathrm{vom} \mathrm{dreiwertigen}$ Vanadin wufsten. Dann stellte Petersen einige Doppelfluoride dar, die sich als isomorph mit den entsprechenden Ferrisalzen erwiesen. Hierauf machte PICCINI die Vanadinalaune. Schliefslich gewann ich die Salze $\mathrm{K}_{3} \mathrm{~V}(\mathrm{CN})_{6}$ und $\mathrm{K}_{3} \mathrm{~V}(\mathrm{CNS})_{6} .4 \mathrm{H}_{2} \mathrm{O}$, dessen Lösung

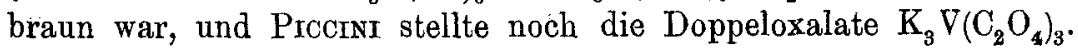
$3 \mathrm{H}_{2} \mathrm{O}$ u. s. w. dar. Wenn man jemandem sagt, dafs ein Element $X$ die folgenden Salze bildet: $\mathrm{K}_{2} \mathrm{XF}_{5}, \mathrm{~K}_{8} \mathrm{X}(\mathrm{CN})_{6}, \mathrm{~K}_{3} \mathrm{X}\left(\mathrm{C}_{2} \mathrm{O}_{4}\right)_{3} .3 \mathrm{H}_{2} \mathrm{O}$, $\mathrm{KX}\left(\mathrm{SO}_{4}\right)_{2}, 12 \mathrm{H}_{2} \mathrm{O}$ und $\mathrm{K}_{3} \mathrm{X}(\mathrm{CNS})_{6}, 4 \mathrm{H}_{2} \mathrm{O}$, von denen das letztere eine sehr charakteristische Farbe zeigt, würde er dann nicht sofort sagen, dals $\mathrm{X}$ dreiwertiges Eisen ist? - Andererseits, als ich einst Vanadin zu seinen zweiwertigen Verbindungsformen reduzieren wollte, benutzte ich eine schwefelsaure Lösung. Damals waren noch 
keine Salze des zweiwertigen Vanadins bekannt. Nach der Reduktion jedoch zeigte sich kein Niederschlag eines unlöslichen Sulfats. Wenn demnach das Vanadin dem Baryum nicht verwandt war, so mufste es mit dem Magnesium Ähnlichkeit zeigen und ein lösliches Sulfat der Formel $\mathrm{VSO}_{4} \cdot 7 \mathrm{H}_{2} \mathrm{O}$ geben. Ich versuchte, dies Salz darzustellen, was mir aber nicht gelang. Später wurde es von Picciñ beschrieben; es besafs die angegebene Formel und gab ein Kaliumdoppelsalz der Zusammensetzung $\mathrm{K}_{2} \mathrm{~V}\left(\mathrm{SO}_{4}\right)_{2} \cdot 6 \mathrm{H}_{2} \mathrm{O}$, in vollkommener Analogie mit Magnesium und Zink.

Wir wollen jetzt betrachten, inwieweit die obigen Thatsachen mit dem periodischen System übereinstimmen, und inwiefern das System sie erklärt. Eine der fundamentalsten Thatsachen der anorganischen Chemie ist es, dafs ein Element mehr als eine Valenzstufe besitzen kann. Das System fordert nur eine einzige Stufe in den ersten vier Familien, und nur zwei für jedes Element in den anderen. Weiter stellt jedes Element, welches mehr als ein Oxyd oder Chlorid zu bilden vermag, einen Widerspruch zu dem System in seiner jetzigen Form dar.

Um zu zeigen, wie wenig überhaupt auf dem Wege der systematischen Prüfung des periodischen Gesetzes geschehen ist, wollen wir zuerst ein sehr einfaches Beispiel nehmen. Wir haben geseben, dals Natriumsalze fast durchweg in Wasser löslich sind, unabhängig von der Natur und Valenz ihrer sauren Radikale. Diese allgemeine Löslichkeit mufs demnach abhängig sein von einer Eigenschaft des Natriumions selbst. Kalium mufs die gleiche Eigenschaft besitzen, denn auch Kaliumsalze sind löslich. Es gehört der gleichen Familie wie das Natrium an und ist etwas schwerer. Wie beeinflufst nun das zunehmende Atomgewicht die allgemeine Löslichkeit? Ist der Einflufs derselbe - oder wenigstens annähernd derselbe - für alle Salze? Oder werden Änderungen in verschiedenen Richtungen hervorgerufen bei Salzen von einbasischen und zweibasischen Säuren, von starken und schwachen Säuren, oder bei normalen und sauren Salzen? Ein Vergleich der Löslichkeiten der Salze des Natriums und des Kaliums allein verneint sofort die erste Frage, bringt aber in die letztere keine Klarheit. Natriumchlorid ist weniger löslich als Kaliumchlorid, Natriumjodid ist löslicher als Kaliumjodid. Das Chlorat und das Perchlorat von Kalium sind wenig löslich, die entsprechenden Natriumsalze sind sehr löslich. Natriumbikarbonat ist wenig löslich, Kaliumbikarbonat ist leicht löslich. Irgend welche Annahme über die Abstufung vom Natrium 
zum Kalium ist deswegen im Lichte solcher Thatsachen gezwungen und willkürlich. Die Frage ist aber eine weitreichende und von fundamentaler Wichtigkeit für das periodische System. Vielleicht - ja sogar wahrscheinlich - existiert eine Abstufung; der einzige Weg aber, auf dem sie aufzufinden ist, besteht darin, dafs man die Löslichkeiten der Rubidium - und Cäsiumsalze prüft. Stellen wir beispielsweise fest, ob die Löslichkeiten des Chlorids, Bromids und Jodids von Cäsium einander näher liegen, als die der Kaliumsalze! Ist dies der Fall, so ist ein wichtiger Anomalie - die Thatsache, dafs die Löslichkeiten von $\mathrm{NaCl}$ und $\mathrm{KCl}$ und von $\mathrm{NaJ}$ und $\mathrm{KJ}$ im umgekehrten Verhältnis stehen aufgeklärt. Wenn dies nicht der Fall ist, so haben wir auch gewonnen; denn wenn wir der Ansicht sind, dafs negativen Resultaten keine Bedeutung zukommt, so würden wir damit sagen, dafs es nicht notwendig wäre, das System, mit dem wir arbeiten, durch Thatsachen zu prüfen.

Ich will noch ein Beispiel gleicher Art anführen, bei dem wir aber bekanntere Daten zum Vergleich haben. Silberjodid ist weniger löslich als das Bromid und dieses wieder weniger als das Chlorid. Beim Natrium wächst die Löslichkeit in der umgekehrten Reihenfolge - Chlorid-Bromid-Jodid. Ebenso ist es mit jedem anderen Metall. Wenn das Chlorid reichlich löslich ist, so ist es um so mehr das Jodid. Ist das Chlorid schwer löslich, so ist das Jodid fast unlöslich. Natrium, Baryum, Kalium, Eisen sind Beispiele für den einen Fall; Silber, Blei, Thallium, Quecksilber sind Beispiele für den anderen. ${ }^{1}$ Wo liegt die Grenzlinie? Es scheint mir, dals eine Regel wie diese sehr leicht die Richtung zu einem allgemeinen Gesetz von fundamentaler Wichtigkeit zeigen kann.

Wenn wir zu der Gruppe der zweiwertigen Metalle kommen, so wird die Erklärung der Thatsachen durch das periodische System - selbst mit hinreichenden Daten - praktisch unmöglich. Wir wollen die Gruppe: $\mathrm{Mg}, \mathrm{Mn}, \mathrm{Fe}, \mathrm{Ni}, \mathrm{Co}, \mathrm{Cu}, \mathrm{Zn}, \mathrm{Cd}$ wählen. Von dieser gehören drei Elemente: $\mathrm{Mg}, \mathrm{Zn}, \mathrm{Cd}$ in die zweite Familie, $\mathrm{Mn}$ in die siebente, $\mathrm{Fe}$, $\mathrm{Co}$ und $\mathrm{Ni}$ in die achte und $\mathrm{Cu}$ in die erste. Wir können noch hinzufügen zweiwertiges Vanadin und Chrom, aus der fünften bezw. der sechsten Familie, so dals wir im ganzen 10 Metalle haben, die sich auf 6 von den 8 Familien verteilen. Wie wir gesehen haben, sind ihre typischen Reaktionen

1 Kohrradscr, Sitzungsber. d. Kgl. Preuss. Akad. d. Wiss. 8, 90-94. Z. anorg. Chem. Bd. 33. 
qualitativ dieselben: Löslichkeit, sowie Bildung von Doppelsalzen und komplexen Aminen. Ihre Verbindungen sind so sehr ähnlich, dals wir für ibre analytische Trennung entweder sehr geringe qualitative Unterschiede in der Löslichkeit verwenden müssen, wie beim Zink - und Cadmiumsulfid, oder dafs wir sie in eine andere Oxydationsstufe überführen müssen, z. B. das Ferroeisen in das Ferrieisen, das Mangan in sein Dioxyd, Kobalt in seine dreiwertige Form, wie im $\mathrm{K}_{3} \mathrm{Co}\left(\mathrm{NO}_{2}\right)_{6}$, Kupfer in seine einwertige Form (CuCNS). Die Frage, ob sich in einer solchen Gruppe Abstufungen aufinden lassen, ist eine sehr weitreichende. Das periodische System kann uns kaum sagen, was zu erwarten ist. In der That wird darin gar nicht die Existenz von Mangano-, Ferro-, Kobalto- und Nickelverbindungen berücksichtigt. Allein die Ähnlichkeit des zweiwertigen Kupfers mit dem Zink kann auf die nahe Stellung der beiden Metalle zurückgeführt werden. - Als Grundlage werden wir deswegen die Magnesium-, Zink- und Cadmiumsalze wählen müssen, welche zu derselben Familie gehören. Bei diesen drei Metallen kann man die Existenz einer Abstufung annehmen. Wie ist nun das relative Verhalten der anderen Elemente. HтTтоRF hat gefunden, dals in konzentrierten Lösungen das Kaliumcadmiumjodid $\mathrm{K}_{2} \mathrm{CdJ}_{4}$ zum grö isten Teile in die Ionen $2 \mathrm{~K}^{\circ}$ und $\mathrm{CdJ}_{4}{ }^{\prime \prime}$ zerfällt; während das entsprechende Kaliumzinkjodid einen vollständigeren Zerfall erleidet, indem in seiner Lösung nur wenige $\mathrm{ZnJ} J_{4}$-Anionen vorhanden sind. Magnesiumdoppelsalze sind sprichwörtlich unbeständig. Hier haben wir in der Stabilität der Doppeljodide eine Abstufung von Magnesium zum Cadmium. Lälst sich diese Abstufung nun auch in der gleichen Ordnung und Richtung bei den anderen Doppelsalzen derselben drei Elemente nachweisen? Die Frage lärst sich heute noch nicht beantworten. Falls eine derartige Abstufung gefunden würde, so fragt es sich, ob sich ihr auch die zwischen Magnesium und Zink liegenden Metalle anschliefsen. Nimmt die Tendenz zur Bildung von Doppelsalzen von einem zum anderen zu mit steigendem Atomgewicht durch die ganze Gruppe? Die Litteratur giebt uns keine Auskunft. Ich habe versucht, die Existenz gewisser Verbindungen als Wegweiser bei der Beantwortung dieser Frage zu verwenden. Z. B. vereinigt sich Cuprichlorid mit Chlorwasserstoffsäure zu der Säure $\mathrm{HCuCl}_{3}$. Als Regel gilt, dals Doppelsalze, deren freie Säure existieren, stabiler sind, als diejenigen, von denen die freie Säure nicht erhalten werden kann. Cupridoppelsalze sollten deswegen verhältnismälsig beständig sein. Solche Schlüsse zu ziehen, würde aber 
gleichbedeutend sein mit der Annahme, dafs ein Körper, der noch nicht beschrieben ist, auch nicht existieren kann, was im vorliegenden Falle wenigstens ziemlich willkürlich wäre. Der einzige Weg, der zur Lösung eines solchen Problemes offen steht, sind physikalisch-chemische Messungen. Leider sind nun exakte Messungen von dieser Art schwierig, besonders dann, wenn sie an Doppelsalzen auszuführen sind, die leicht molekularer Dissoziation unterliegen, und bei denen sehr konzentrierte Lösungen verwendet werden müssen. Selbst bei verdünnten Lösungen ist wegen der komplizierten Bedingung die Gefrierpunkts - und die Leitfähigkeitsmethode u. s. w. etwa nur auf $5 \%$ genau, und diese Fehlergrenze ist für vergleichende Messungen zu grofs.

Nichtsdestoweniger lassen sich Methoden auffinden, die zwar keine absoluten Werte ergeben, die aber doch wenigstens einen Vergleich ihrer Resultate gestatten. In einem Falle ist dies sehr hübsch ausgearbeitet, indem einfach die. Menge des überschüssigen Cyankaliums bestimmt wurde, die erforderlich war, um die Fällung von Zinksulfid aus einer Lösung von Kaliumzinkcyanid zu verhindern. Solch eine Methode, gleichförmig auf alle Cyanide der Gruppe angewendet, würde uns - vorausgesetzt, dafs die Löslichkeit der Sulfide selbst bekannt wäre, ein direktes Mafs - ausgedrückt in den Quantitäten Kaliumcyanid - geben für die Stabilität dieser Doppelsalze. Die Einheit wäre zwar willkürlich, aber wir würden wenigstens einen Angriffspunkt besitzen. - Würde nun die Reihenfolge der Stabilität bei den Doppelcyaniden dieselbe sein wie bei den Doppeljodiden? Würde auch die relative Stabilität der Doppelchloride dieselbe sein?

Hieran schliefst sich die weitere Frage, ob Jod eine wesentlich gröfsere Tendenz zum Eintritt in komplexe Anionen hat als das Chlor, oder umgekehrt. Darüber haben wir heute nur sich wiedersprechende Angaben. Nach weiterer Erforschung wird sich aber wahrscheinlich ergeben, dafs in der Stabilität der Doppelchloride und Doppeljodide eine ähnliche Beziehung besteht, wie oben bei der Löslichkeit der einfachen Haloidsalze erwähnt wurde. Eine Andeutung, daís dies der Fall ist, sieht man schon aus den - rein qualitativ bekannten - Stabilitätsverhältnissen der Doppelbaloidsalze der Metalle der zweiten Familie des Systemes. Aus verschiedenen Reaktionen geht hervor, dafs an dem einen Ende der Reihe Merkuridoppeljodide beständiger sind als die entsprechenden Chloride. Beim Zink dagegen ist offenbar zwischen den beiden Körpern nur ein geringer Unterschied. Beim Magnesium sind alle 
Doppelsalze sehr wenig beständig und so bilden sie das andere Ende der Reihe, wo die Doppelchloride am beständigsten sind. Ein Cäsiummagnesiumjodid ist überhaupt nicht darstellbar gewesen. ${ }^{1}$

Allgemein ist jetzt die Anschauung durchgedrungen, dals die Löslichkeit eines Salzes wie Silberchlorid in Ammoniak auf die Bildung eines komplexen Kations zurückzuführen ist, dals seine Löslichkeit in einer Lösung des entsprechenden Alkalisalzes auf Bildung eines komplexen Anions beruht, wobei das Alkalimetall zum Kation wird. Ferner nimmt man an, dals der Unterschied zwischen „Doppelsalzen" und „Salzen komplexer Säuren" lediglich ein gradueller Unterschied in der Stabilität ist und nicht eine qualitative Verschiedenheit. Die Formeln dieser Verbindungen und die Frage, ob ein gegebenes Element dieselben bildet, dient zur Charakterisierung eines solchen Elementes und zur Einreihung desselben in diese oder jene Gruppe von Elementen derselben Valenz. So weit sind nur qualitative Daten erforderlich. Um aber die definitive relative Stellung eines Elementes in seiner Gruppe festzulegen, bedürfen wir quantitativer Messungen, wie sie die Litteratur bis jetzt noch nicht enthält. DAwson und $\mathrm{MCCRAE}^{2}$ haben z. B. gefunden, dafs Lösungen der Sulfate von Kupfer, Nickel, Zink und Cadmium in Ammoniak alle komplexe Verbindungen der allgemeinen Formel $\mathrm{M}^{\prime \prime}\left(\mathrm{NH}_{3}\right)_{\mathbf{4}} \mathrm{SO}_{4}$ enthalten. Der Einflufs einer Änderung des Atomgewichtes des Metalles $\mathrm{M}$ ist also in den Formeln der Verbindungen nicht zum Ausdruck gekommen, und kann nur bestimmt werden durch Messung der relativen Werte der Dissoziationskonstanten dieser Salze, oder durch Messung solcher Eigenschaften, die der Reihenfolge und dem Werte nach dieser Gröfse parallel gehen. Heute wissen wir kaum, ob die Natur des sauren Radikals einen Einflufs auf die Gleichgewichtsverhältnisse in der Lösung der Metallamine hat. Wenn dies nicht der Fall ist, so mufs es möglich sein, die Tendenz des zweiwertigen Kupfers und des Nickels zum Eintritt in komplexe Kationen bezogen auf die gleiche Tendenz des Zinks zum Ausdruck zu bringen. Hat das negative Radikal aber einen derartigen Einflufs, würde dann andererseits die relative Stabilität eines Kupfersulfatkomplexes gegenüber dem Chloridkomplex ebenso grofs sein, wie bei den Zinkrerbindungen? Solche Fragen können angenähert beantwortet werden, indem man lediglich Lösungen

1 Wells und Campbeux, 2. anorg. Chem. 5, 272.

2 Z. anorg. Chem. 26, 99. 
der einfachen Salze in äquivalenten Verdünnungen mit Ammoniak titriert, bis sich die ausgefällten Hydroxyde wieder lösen.

Ich habe versucht, einige Probleme von dieser allgemeinen Natur durch Studium der Löslichkeit isomorpher Doppelsalze in Wasser bei konstanter Temperatur zu lösen. Doppelsalze wurden lediglich deswegen gewählt, weil ihre Löslichkeit in weiten Grenzen variiert, und weil sie nicht nur von Alkalimetallen gebildet werden, sondern auch von Thallium, das nicht der ersten Familie des Systems angehört, und von dem Radikal Ammonium. Der Einflufs des einwertigen Metalles konnte so auf breiter Basis studiert werden. Zuerst wurden die Alaune untersucht. Diese wurden gewählt, weil unter den dreiwertigen Elementen, die Alaune bilden, sich das Vanadin befindet, dessen Verbindungen vom Typus $V X_{3}$ eine wenig beständige Oxydationsstafe darstellen; hier erhebt sich nun die Frage, ob dieser Umstand das Verhalten der Salze beeinflufst. Die Löslichkeiten der Alaune von Aluminium, Vanadin, Chrom und Eisen mit Cäsium, Rubidium, Thallium und Ammonium bei $25^{\circ}$ sind weiterhin angegeben und zwar ausgedrückt in Grammmolekülen pro Liter. ${ }^{1}$

$\begin{array}{lcccc} & \mathrm{Al} & \mathrm{Cr} & \mathrm{V} & \mathrm{Fe} \\ \mathrm{Cs} & 0.013 & 0.015 & 0.020 & 0.045 \\ \mathrm{Rb} & 0.059 & 0.078 & 0.177 & 0.293 \\ \mathrm{Tl} & 0.177 & 0.212 & 0.573 & 0.799 \\ \mathrm{NH}_{4} & 0.387 & 0.407 & 1.210 & 1.659\end{array}$

Zunächst bemerkt man, dafs diese Zahlen weder die gleiche Reihenfolge wie die Atomgewichte der einwertigen Metalle, noch die der dreiwertigen Elemente zeigen. Trotzdem läist sich der Einflufs jedes Elementes leicht erkennen, indem die Löslichkeiten regelmälsig sowohl in vertikaler als horizontaler Richtung wachsen.

Die Beziehungen zwischen den verschiedenen Werten kann am besten dargestellt werden, wenn man die Löslichkeiten als Ordinaten und die Atomgewichte des dreiwertigen Metalles als Abscissen wählt (Fig. 1).

Jede derartige gebrochene Linie giebt die Beziehungen an zwischen den Alaunen verschiedener dreiwertiger Metalle bei gegebenem einwertigen Metall. In einer anderen Mitteilung war der

1 Am. Chem. Journ. 26, 166.

${ }^{2}$ Am. Chem. Journ. 26, 332. 
Versuch gemacht worden, zu zeigen, dafs alle Linien, die die Alaune zweier dreiwertiger Metalle mit korrespondierenden Alkalimetallen verbinden, einen gemeinsamen Schnittpunkt haben; es war auch für die Löslichkeiten eine allgemeine Formel auf dieser Basis berechnet worden.

Wenn wir die Differenz der Löslichkeiten der Alaune eines dreiwertigen Metalles mit zwei Alkalimetallen als Inkrement der Löslichkeit für die letzteren bezeichnen, so sollte hiernach das Verhältnis zwischen den Löslichkeitsinkrementen der Alaune zweier dreiwertiger Metalle für korrespondierende Alkalimetalle konstant sein. Nachdem der Fehler in den beobachteten Löslichkeiten empirisch aus.

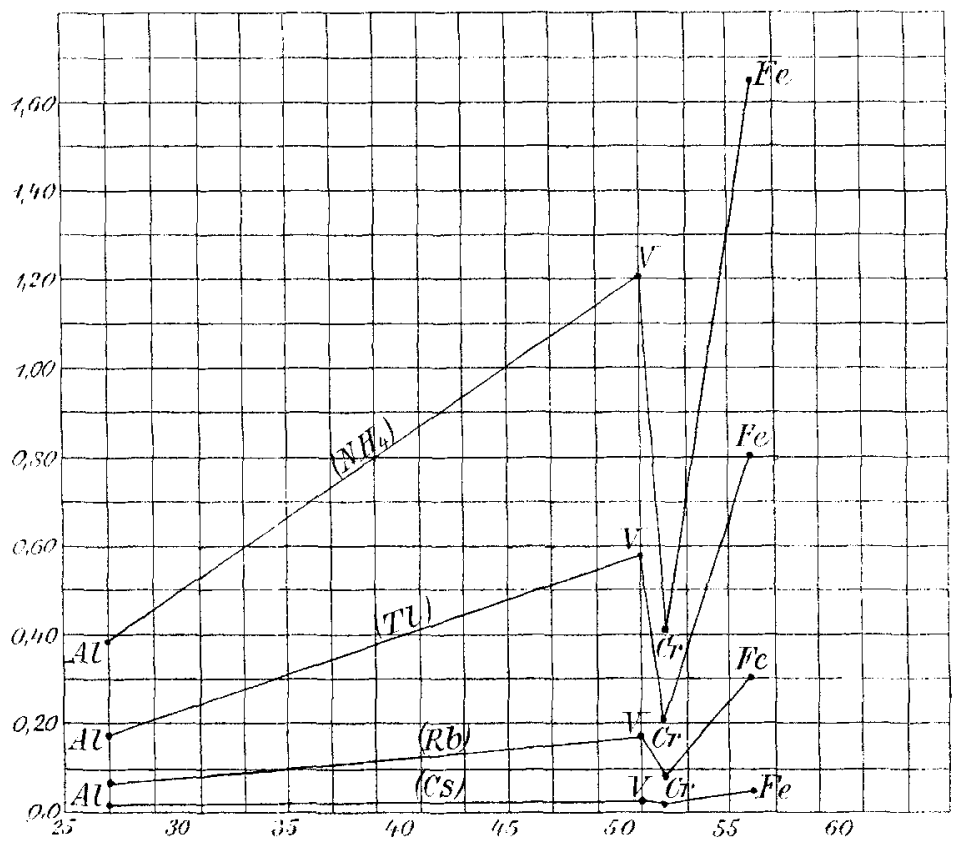

Fig. 1.

Die Abscissen stellen die Atomgewichte, die Ordinaten die Löslichkeiten dar. Die Buchstaben in Klammern geben die im Alaun enthaltenen einwertigen Elemente an. Jede Kurve giebt also die Beziehungen zwisehen den Alaunen eines gegebenen einwertigen Elementes mit den verschiedenen dreiwertigen Metallen an.

geglichen war, wurde als theoretisches Verhältnis der Inkremente $\frac{V m_{2}-m_{1}}{A l m_{2}-m_{1}}$ berechnet 3.21. Gefunden wurden die folgenden Werte: 


$\begin{array}{lcc}m_{2} & m_{1} & \text { Verhältnis } \\ \mathrm{NH}_{4} & \mathrm{Cs} & 3.18 \\ \mathrm{Tl} & \mathrm{Cs} & 3.38 \\ \mathrm{Rb} & \mathrm{Cs} & 3.40 \\ \mathrm{NH}_{4} & \mathrm{Rb} & 3.15 \\ \mathrm{Tl} & \mathrm{Rb} & 3.36 \\ \mathrm{NH}_{4} & \mathrm{Tl} & \mathbf{3 . 0 3}\end{array}$

Die Differenzen zwischen diesen Zahlen und dem berechneten Werte sind nicht grö Iser, als sie durch einen mittleren Fehler von nicht mehr als 0.005 Gramm-Mol. hervorgerufen werden. Seitdem hat sich jedoch gezeigt, dals die Abweichungen der gefundenen Schnittpunkte von dem gemeinschaftlichen Punkt zwar gering, aber regelmälsig sind, und dals die verschiedenen Linien Tangenten an eine schmale Hyperbel darstellen. Die mathematische Entwickelung dieser Angelegenheit ist anderswo entwickelt worden. ${ }^{1}$ Es hat sich gezeigt, dals die Löslichkeiten sich sehr genau ausdrücken lassen durch die Gleichung:

$$
Y=a y^{2}+2 b y+c
$$

in der $Y$ die Löslichkeit eines Alauns eines gegebenen Alkalimetalles mit dem einen dreiwertigen Metall und $y$ die Löslichkeit des Alauns mit einem anderen dreiwertigen Metalle ist. Die Werte der Konstanten $a, b$ und $c$ sind gegeben durch die Gleichungen

$$
\begin{aligned}
& a=\frac{\frac{Y-Y_{1}}{y-y_{1}}-\frac{Y_{1}-Y_{2}}{y_{1}-y_{2}}}{y-y_{2}} \\
& 2 b=\frac{Y-Y_{1}}{y-y_{1}}-a\left(y-y_{1}\right) \\
& c=Y-y \frac{Y-Y_{1}}{y-y_{1}}+a y y,
\end{aligned}
$$

in denen $y, y_{1}, y_{2}$ die Löslichkeiten der Alaune eines gegebenen dreiwertigen Metalles mit verschiedenen einwertigen Metallen, nach ihren Grölsen geordnet, bedeuten. Die Werte für die drei Konstanten der Aluminium-Vanadinreihe - berechnet für alle möglichen Werte von $y, y_{1}$, u. s. w. - sind in der folgenden Tabelle zusammengestellt. Unter $i$ sind die beobachteten und berechneten Verhältnisse der Inkremente angegeben. Der Wert $e$, welcher die Löslichkeit $Y$ für $y=0$ angiebt, wird in $1 / 1000 \mathrm{~g}$-Mol. ausgedrückt.

1 Am. Chem. Journ. 27, 455. 


\begin{tabular}{|c|c|c|c|c|c|c|c|}
\hline$y$ & $y_{1}$ & $y_{8}$ & $a$ & $2 b$ & $c$ & $\begin{array}{c}i \\
\text { berechn. }\end{array}$ & $\begin{array}{c}i \\
\text { beobacht. }\end{array}$ \\
\hline $\mathrm{NH}_{4}$ & $\mathrm{Tl}$ & $\mathrm{Rb}$ & -0.0009 & 3.54 & - & - & - \\
\hline $\mathrm{NH}_{4}$ & $\mathbf{T l}$ & Cs & $-0.0009 *$ & - & -26.2 & 3.03 & 3.03 \\
\hline $\mathrm{NH}_{4}$ & $\mathrm{Rb}$ & $\mathrm{Cs}$ & -0.0008 & 3.55 & -29.0 & 3.14 & 3.15 \\
\hline $\mathrm{TI}$ & $\mathrm{Rb}$ & $\mathrm{Cs}$ & -0.0007 & 3.56 & -29.4 & 3.33 & 3.35 \\
\hline $\mathrm{NH}_{4}$ & Cs & - & - & $3.54^{*}$ & $-25.2^{*}$ & 3.18 & 3.18 \\
\hline $\mathrm{Tl}$ & $\mathrm{Cs}$ & - & - & 3.52 & -29.1 & 3.37 & 3.39 \\
\hline $\mathrm{Rb}$ & $\mathrm{Cs}$ & - & - & 3.41 & -24.3 & 3.47 & 3.41 \\
\hline
\end{tabular}

Die von experimentellen Fehlern am wenigsten beeinflufsten Werte sind durch Sterne gekennzeichnet. Unter Anwendung dieser Konstanten erhält man die Gleichung

$$
Y=3.54 y-0.0009 y^{2}-25.2 \text {. }
$$

Die Beziehungen zwischen den Löslichkeiten der Aluminiumalaune und denen der anderen dreiwertigen Metalle werden ebenso deutlich ausgedrückt durch dieselbe allgemeine Gleichung mit anderen Werten für $a, b$ und $e$; es sind die folgenden:

$$
\begin{aligned}
& \text { Cr: } Y=1.37 y-0.0008 y^{2}-2 \\
& \text { Fe: } Y=4.84 y-0.0013 y^{2}-18.8 \text {. }
\end{aligned}
$$

Die folgende Tabelle enthält die Löslichkeiten der Vanadin-, Chrom- und Eisenalaune, berechnet aus diesen Formeln unter der Annahme, dafs die für die Aluminiumsalze bestimmten Werte absolut korrekt sind. Unter $D$ sind die Abweichungen von den beobachteten Löslichkeiten angegeben.

\begin{tabular}{lcrcccc} 
& $\mathrm{V}$ & \multicolumn{1}{c}{$D$} & $\mathrm{Cr}$ & $D$ & $\mathrm{Fe}$ & \multicolumn{1}{c}{$D$} \\
$\mathrm{Cs}$ & 0.020 & 0.000 & 0.015 & 0.000 & 0.045 & 0.000 \\
$\mathrm{Rb}$ & 0.180 & +0.003 & 0.076 & -0.002 & 0.262 & -0.031 \\
$\mathrm{Tl}$ & 0.573 & 0.000 & 0.215 & +0.003 & 0.797 & -0.002 \\
$\mathrm{NH}_{4}$ & 1.211 & +0.001 & 0.408 & +0.001 & 1.659 & 1.000
\end{tabular}

Ersetzt man in den obigen Gleichungen $y$ durch das Verhältnis $y \frac{A l, m^{I}}{A l,} \overline{C s}=m y$, so erhält man die Gleichung in der Form:

$$
Y=a m^{2} y^{2}+2 b m y+c .
$$

Da für jedes Alkalimetall $m$ einen anderen Wert hat und da die Konstanten $a, b$ und $c$ nur von dem dreiwertigen Metall ab- 
hängen, so heifst das, dafs wir die Löslichkeit irgend eines der 16 Alaune direkt aus der des Aluminium-Cäsiumsalzes erhalten können, indem wir einfach in die Gleichung die den einwertigen und den dreiwertigen Metallen der Verbindungen zugehörigen Konstanten einsetzen.

Hierdurch ist eines der oben angedeuteten Probleme, wenigstens in einer seiner Phasen gelöst. Der relative Einflufs eines gegebenen Elementes auf die Löslichkeit der Verbindungen in einer isomorphen Reihe kann durch eine Konstante dargestellt werden, die unverändert bleibt durch alle Glieder der Reihe, die sie bildet. Es macht keinen Unterschied, ob das Element in derselben Reihe des periodischen Systemes steht, oder in einer anderen; ob seine Valenz die gewöhnliche für das Element oder eine abnormale ist; oder ob schliefslich an Stelle eines Elementes eine Atomgruppe tritt. - Wenn dies für die Löslichkeiten zutrifft, kann es denn nicht auch für andere Eigenschaften richtig sein? Je genauer man ein vergleichendes Studium der anorganischen Chemie durchführt, um so klarer wird dies werden, weil eine grofse Reihe von Regelmälsigkeiten heute nur oberflächlich und nach ihrer qualitativen Seite hin bekannt sind. Die Aufmerksamkeit ist in der That davon abgelenkt worden, weil man zu sehr am periodischen System als einziger Basis der Klassifikation festhielt. Die Regelmälsigkeiten werden durch das System nicht erklärt, vielmehr stehen sie oft mit der jetzigen Form desselben in Widerspruch, da die Fälle, dafs Elemente, die isomorphe Salze bilden, zu verschiedenen Familien gehören, häufiger vorkommen, als die entgegengesetzten. Sicherlich aber ist die anorganische Chemie noch nicht erschöpft, solange diese Regelmälsigkeiten noch nicht hinreichend studiert sind.

Die nächste Reihe, die ich untersucht habe, waren die Verbindungen vom allgemeinen Typus $\mathrm{M}_{2}{ }^{\mathrm{I}} \mathrm{M}^{\mathrm{I}}\left(\mathrm{SO}_{4}\right)_{2} \cdot 6 \mathrm{H}_{2} \mathrm{O} . \mathrm{M}^{\mathrm{I}}$ ist irgend ein Alkalimetall - ausgenommen Natrium - oder Thallium und Ammonium. M山 entspricht Magnesium, Mangan, Ferroeisen, Nickel, Kobalt, Kupfer, Zink und Cadmium. Theoretisch sind 40 verschiedene Salze möglich; leider aber werden manche der Thalliumverbindungen und auch einige der Kaliumsalze durch Wasser zersetzt. Die vollständigen Resultate dieser Untersuchung sind in dem American Chemical Journal veröffentlicht worden ${ }^{\mathfrak{l}}$ und ich

$127,455$. 
werde mich hier auf eine Aufzählung der wichtigsten Punkte beschränken.

Drei allgemeine Fragen waren zu beantworten: 1. Ist die Reihenfolge der Löslichkeiten korrespondierender Salze verschiedener zweiwertiger Metalle dieselbe, wie die Reihe der Atomgewichte der letzteren? 2. Entsprechen die Löslichkeiten hier demselben oder einem ähnlichen mathematischen Gesetz wie bei den Alaunen? 3. Kommen die Salze der Alkalimetalle in die gleiche Reihenfolge wie dort? Natürlich war nicht zu erwarten, dafs die Werte für die Konstanten der Alkalimetalle dieselben sein würden wie oben, aber die Frage, ob der allgemeine Einfluís eines gegebenen Alkalimetalles auf seine Doppelsalze in verschiedenen Reihen ähnlich ist, hat allgemeine Bedeutung.

Vergleicht man die Löslichkeiten der Cäsiumdoppelsalze der sämtlichen zweiwertigen Metalle, so sieht man, dafs durchaus keine Konformität mit den Atomgewichten der letzteren besteht. Es folgen hier die Löslichkeiten dieser Salze bei $25^{\circ}$ in Grammmolekülen pro Liter Wasser.

$\begin{array}{llllllll}\text { Ni 58.7 } & \text { Zn 65.4 } & \text { Co } 59 & \text { Cu 63.6 } & \text { Mg 24 } & \text { Mn 54 } & \text { Fe } 56 & \text { Cd } 112\end{array}$ $\begin{array}{llllllll}0.495 & 0.738 & 0.810 & 0.882 & 1.106 & 1.570 & 1.967 & 2.455 .\end{array}$

Selbst die Glieder derselben Familie im System zeigen keine Regelmälsigkeit. Die Zinksalze sind weniger löslich als die des Magnesiums und Cadmiums; die Nickelsalze weniger als die des Eisens und Kobalts. Das Gleiche gilt für die Reihenfolge, welche die Alkalimetalle einnehmen. Bei den Alaunen war die Ordnung:

$$
\mathrm{Cs}, \mathrm{Rb}, \mathrm{Tl}, \mathrm{NH}_{4}, \mathrm{~K} \text {. }
$$

Andererseits sind die Löslichkeiten der Nickelverbindungen folgende:

$\begin{array}{ccccc}\mathrm{Tl} & \mathrm{Rb} & \mathrm{K} & \mathrm{NH}_{4} & \mathrm{Cs} \\ 0.070 & 0.142 & 0.209 & 0.264 & 0.495 .\end{array}$

Die Rubidiumsalze sind weniger löslich als die des Kaliums; und an den beiden Enden der Reihe haben wir Thallium und Cäsium, die Elemente mit den relativ gröfsten Atomgewichten. Daraus scheint hervorzugehen, dafs überhaupt keine Beziehung zwischen Löslichkeit und Atomgewicht besteht.

Nichtsdestoweniger besteht hier dieselbe mathematische Beziehung wie bei den Alaunen. Es folgen hier die Löslichkeiten 
der Nickel-, Kobalt-, Kupfer - und Zinksalze; dies sind leider die einzigen, die für vier und mehr Alkalimetalle gemessen werden konnten.

$\begin{array}{lcccc} & \mathrm{Ni} & \mathrm{Co} & \mathrm{Cu} & \mathrm{Zn} \\ \mathrm{Tl} & 0.070 & - & 0.122 & 0.129 \\ \mathrm{Rb} & 0.142 & 0.220 & 0.241 & 0.236 \\ \mathrm{~K} & 0.209 & 0.391 & 0.350 & 0.393 \\ \mathrm{NH}_{4} & 0.264 & 0.513 & - & 0.480 \\ \mathrm{Cs} & 0.495 & 0.810 & 0.882 & 0.738\end{array}$

Nimmt man die Nickelsalze, als die am wenigsten löslichen zur Basis, so ergeben sich als Werte $a, b$ und $c$ für alle verschiedenen Werte von $y, y_{1}$ und $y_{2}$ und als Verhältnisse der Inkremente $(i)$ die folgenden Zahlen.

Nickel - Kobalt.

\begin{tabular}{l|l|l|l|c|c|c|c}
\hline$y$ & $y_{1}$ & $y_{2}$ & $a$ & $2 b$ & $c$ & $\begin{array}{c}\text { beob. } \\
i\end{array}$ & $\begin{array}{c}\text { berechn. } \\
i\end{array}$ \\
\hline $\mathrm{Cs}$ & $\mathrm{NH}_{4}$ & $\mathrm{Rb}$ & $-0.00320^{*}$ & $3.70^{*}$ & $-243^{*}$ & 1.67 & 1.66 \\
$\mathrm{Cs}$ & $\mathrm{NH}_{4}$ & $\mathrm{~K}$ & -0.00325 & - & - & - & - \\
$\mathrm{Cs}$ & $\mathrm{K}$ & $\mathrm{Rb}$ & -0.00313 & 3.70 & -244 & 1.46 & 1.45 \\
$\mathrm{NH}_{4}$ & $\mathrm{~K}$ & $\mathrm{Rb}$ & -0.00300 & 3.72 & -247 & 1.26 & 1.27 \\
$\mathrm{Cs}$ & $\mathrm{Rb}$ & - & - & $3.70^{*}$ & -239 & 2.40 & 2.40 \\
$\mathrm{NH}_{4}$ & $\mathrm{Rb}$ & - & - & 3.70 & -240 & 2.55 & 2.58 \\
$\mathrm{~K}$ & $\mathrm{Rb}$ & - & - & 3.80 & -237 & 2.22 & 2.19
\end{tabular}

Nickel - Kupfer.

\begin{tabular}{l|l|l|c|c|c|c|c}
\hline$y$ & $y_{1}$ & $y_{\mathbf{g}}$ & $a$ & $2 b$ & $e$ & $\begin{array}{c}\text { beob. } \\
i\end{array}$ & $\begin{array}{c}\text { berechn. } \\
i\end{array}$ \\
\hline $\mathrm{Cg}$ & $\mathrm{K}$ & $\mathrm{Tl}$ & $+0.0005^{*}$ & 1.52 & 12 & 1.86 & 1.87 \\
$\mathrm{Cs}$ & $\mathrm{K}$ & $\mathrm{Rb}$ & +0.0005 & - & - & - & - \\
$\mathrm{K}$ & $\mathrm{Rb}$ & $\mathrm{Tl}$ & +0.0002 & 1.51 & 14 & 1.68 & 1.69 \\
$\mathrm{Cs}$ & $\mathrm{Rb}$ & $\mathrm{Tl}$ & +0.0005 & 1.51 & 16 & 1.82 & 1.83 \\
$\mathrm{Cs}$ & $\mathrm{Tl}$ & - & - & $1.52^{*}$ & $13^{*}$ & 1.79 & 1.80 \\
$\mathrm{Rb}$ & $\mathrm{Tl}$ & - & - & 1.54 & 13 & 1.65 & 1.63 \\
$\mathrm{~K}$ & $\mathrm{Tl}$ & - & - & 1.50 & 15 & 1.64 & 1.65
\end{tabular}


Nickel - Zink.

\begin{tabular}{l|l|l|c|c|c|c|c}
\hline$y$ & $y_{1}$ & $y_{2}$ & $a$ & $2 b$ & $e$ & $\begin{array}{c}\text { beob. } \\
i\end{array}$ & $\begin{array}{c}\text { berechn. } \\
i\end{array}$ \\
\hline $\mathrm{Cs}$ & $\mathrm{NH}_{4}$ & $\mathrm{~K}$ & -0.0016 & 2.33 & 25.5 & 1.12 & 1.12 \\
$\mathrm{Cs}$ & $\mathrm{NH}_{4}$ & $\mathrm{Tl}$ & $-0.0016^{*}$ & - & - & - & - \\
$\mathrm{Cs}$ & $\mathrm{K}$ & $\mathrm{Tl}$ & -0.0016 & 2.33 & 26.5 & 1.20 & 1.20 \\
$\mathrm{NH}_{4}$ & $\mathrm{~K}$ & $\mathrm{Tl}$ & -0.0015 & 2.34 & 25.4 & 1.58 & 1.57 \\
$\mathrm{Cs}_{5}$ & $\mathrm{Tl}$ & - & - & $2.33^{*}$ & $25.5^{*}$ & 1.43 & 1.43 \\
$\mathrm{NH}_{4}$ & $\mathrm{Tl}$ & - & - & 2.33 & 26.4 & 1.80 & 1.81 \\
$\mathrm{~K}$ & $\mathrm{Tl}$ & - & - & 2.34 & 27.4 & 1.90 & 1.89
\end{tabular}

Die Abweichungen liegen überall innerhalb der Fehlergrenzen; dies erkennt man aus dem Vergleich der für $i$ beobachteten und berechneten Werte, bei denen die zweite Dezimale auf eine oder zwei Einheiten unsicher ist.

Wählt man wie früher die durch Sterne bezeichneten Konstanten, so erhält man:

$$
\begin{aligned}
& Y_{\mathrm{Co}}=3.7 y-0.0032 y^{2}-243 \\
& Y_{\mathrm{Cu}}=1.52 y+0.0005 y^{2}+13 \\
& Y_{\mathrm{Zn}}=2.33 y-0.0016 y-25.5
\end{aligned}
$$

Die Werte $\frac{\mathrm{Ni}, \mathrm{M}^{\mathrm{I}}}{\mathrm{Ni}, \mathrm{Cs}}$ sind:

$$
\begin{array}{cccccc}
\mathrm{M}= & \mathrm{Cs} & \mathrm{NH}_{4} & \mathrm{~K} & \mathrm{Rb} & \mathrm{TI} \\
1.0 & 0.533 & 0.422 & 0.287 & 0.141 .
\end{array}
$$

Setzt man diese ein, so erhält man die Löslichkeiten aller Verbindungen dieser Reihe direkt abgeleitet aus der des Cäsium-Nickelsulfats. Diese berechneten Werte sind in der folgenden Tabelle zusammengestellt; die Kolumnen $D$ enthalten die Differenzen gegen die beobachteten Löslichkeiten.

$\begin{array}{lcccccc} & \text { Co } & D & \text { Cu } & D & \text { Zn } & D \\ \text { Tl } & - & - & 0.122 & 0.000 & 0.130 & +0.001 \\ \text { Rb } & 0.222 & +0.002 & 0.239 & -0.002 & 0.273 & +0.037 \\ \text { K } & \mathbf{0 . 3 9 4} & +0.003 & 0.352 & +0.002 & 0.392 & +0.001 \\ \mathrm{NH}_{4} & \mathbf{0 . 5 1 5} & +0.002 & - & - & 0.478 & -0.002 \\ \text { Cs } & \mathbf{0 . 8 0 8} & -0.002 & 0.887 & +0.005 & 0.738 & 0.000\end{array}$

Die einzige Differenz, die aufserhalb der Fehlergrenzen liegt, tritt beim Rubidiumzinksulfat auf; aber auch diese ist nur gering. 
Dafs das Gesetz im allgemeinen richtig ist, geht schon daraus hervor, dafs ich die Löslichkeiten mehrerer Salze dieser Reihe vorhersagen konnte, bevor sie bestimmt waren.

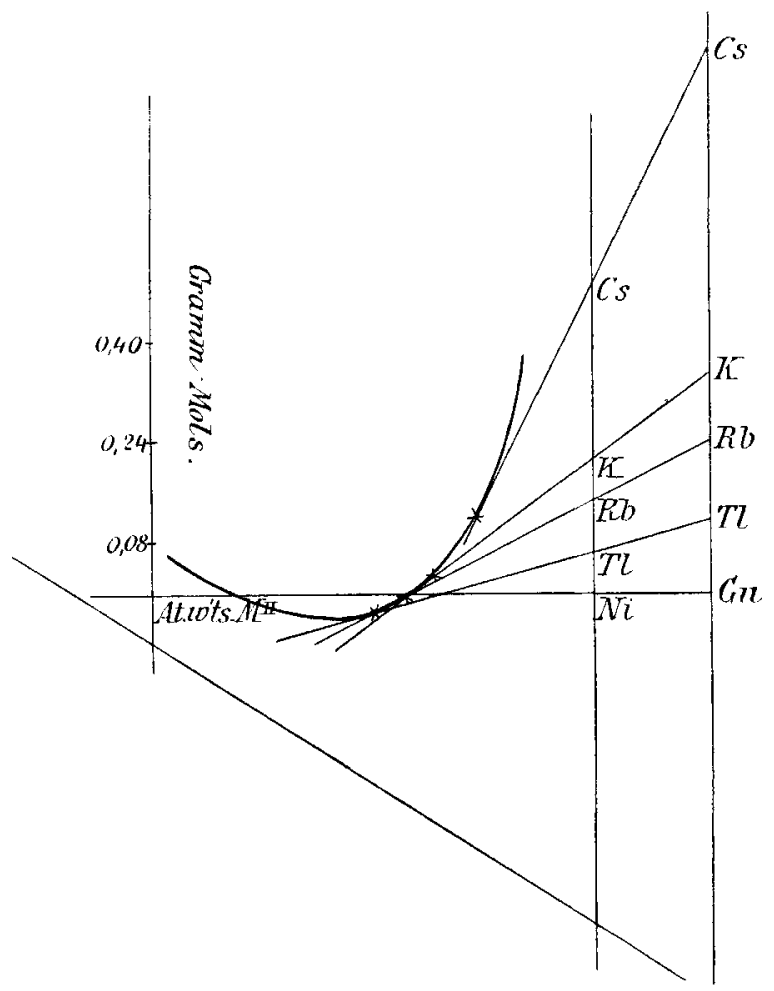

Fig. 2.

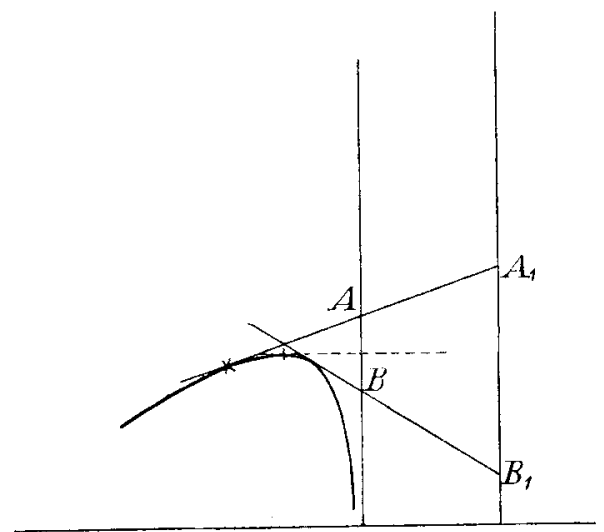

Fig. 3.

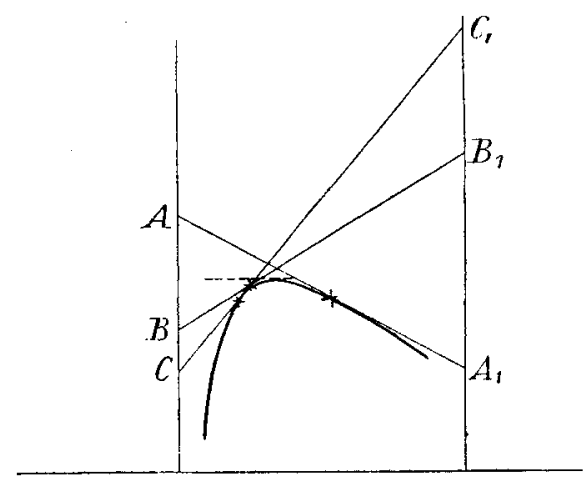

Fig. 4. 
Die Hyperbeln, an welche die Verbindungslinien der Löslichkeitspunkte von Salzen desselben Alkalimetalles mit zweiwertigen Metallen Tangenten darstellen, sind sehr schmal; sie haben als eine Asymptote eine Senkrechte zur $x$-Axe, errichtet in dem Punkte, wo der $x$-Wert für Nickel liegt. Fig. 2 zeigt ibre Lage für die Nickel-Kupferreihe.

Eine nähere Betrachtung dieser Figur lärst einen wichtigen Punkt erkennen: In derselben liegen alle Berührungspunkte an derselben Seite des Berührungspunktes der Horizontalangente. Die verschiedenen Linien gehen also alle nach oben, d. h. die Löslichkeit eines jeden Kupfersalzes ist gröfser als die des entsprechenden Nickelsalzes. Wenn andererseits die Lage der Hyperbel so wäre, dafs die Berührungspunkte der Verbindungslinien extremer Löslichkeitspunkte $\left(A A_{1}-B B_{1}\right)$ an verschiedenen Seiten der Horizontaltangente lägen, so könnten zwei verschiedene Fälle eintreten, deren Betrachtung manche scheinbaren Ausnahmen bei den jetzt üblichen Systematisierungsmethoden aufklären kann. Zunächst liegt bei Fig. 3 die Hyperbel aufserhalb der beiden Senkrechten. Die Löslichkeitsextreme für die Verbindungen des einen Metalles werden in dem einen Falle gröfser $\left(A<A_{1}\right)$, in dem an deren kleiner sein $\left(B>B_{1}\right)$ als die des anderen. Wenn weiterhin die Hyperbel zwischen den zwei Senkrechten liegt (Fig. 4), so wird die Reihenfolge der Löslichkeiten umgekehrt. Der letztere Fall ist mit Sicherheit noch nicht beobachtet worden. Der erstere tritt in dieser Reihe auf und zwar bei dem Zink und Kupfer, sowie bei den Zink- und Kobaltverbindungen.

$\begin{array}{llll}A \cdot \mathrm{Zn}-\mathrm{Cs} & 0.738 & A_{1} \cdot \mathrm{Cu}-\mathrm{Cs} & 0.882 \\ B \cdot \mathrm{Zn}-\mathrm{Tl} & 0.129 & B_{1} \cdot \mathrm{Cu}-\mathrm{Tl} & 0.122 \\ A \cdot \mathrm{Zn}-\mathrm{Cs} & 0.738 & A_{1} \cdot \mathrm{Co}-\mathrm{Cs} & 0.810 \\ B \cdot \mathrm{Zn}-\mathrm{Rb} & 0.236 & B_{1} \cdot \mathrm{Co}-\mathrm{Rb} & 0.220\end{array}$

Allein betrachtet, stehen diese Zahlen im äufsersten Widerspruch zu einander; sie scheinen jede Möglichkeit, die anorganische Chemie jemals zu einer systematisierten Wissenschaft zu machen, zu vernichten. Gerade auf Grund solcher Thatsachen, von denen die Litteratur voll ist, sind bisher allgemeinere Probleme der oben angegebenen Art unbearbeitet geblieben. Diese Werte aber stellen nur bestimmte Punkte in einem allgemeinen Schema dar. Sie können aber interpretiert und als zum Schema gehörig nur erkannt werden, wenn einige der anderen Punkte bereits bestimmt sind. 
Bis jetzt sind vergleichbare Messungen selten an mehr als zwei bis drei Gliedern einer Reihe ausgeführt worden; das Resultat war, dafs scheinbare Unregelmälsigkeiten, wie die obige häufig und deutlich zu Tage traten. Die angeführten Ergebnisse zeigen jedoch an, dafs sie alle aufgeklärt werden können, jedoch nur durch andauernde und sorgfältige Untersuchung ausgedehnter Verbindungsreihen. Die analogen Elemente oder Atomgruppen in isomorphen Verbindungen können als einfache Variable betrachtet werden. Aber gerade wie in jedem anderen Falle müssen wir für jede dieser Variablen so viel Werte wie möglich haben, um ihre Natur sicher stellen zu können.

Dieser Punkt hat, oder kann wenigstens einen entschiedenen Einflufs auf die Bedeutung des mit Rücksicht auf das Atomgewicht abnormalen Verhaltens der Salze der studierten Metalle haben. Wir haben gesehen, dafs die Reihenfolge der Atomgewichte nicht übereinstimmt mit der Reihenfolge der Löslichkeiten. Dann aber ist die Reihenfolge, der die Salze der verschiedenen zweiwertigen Metalle beim Thallium oder Kalium folgen, nicht die gleiche wie beim Ammonium oder Cäsium.

$\begin{array}{lllll} & \text { Ni } & \text { Co } & \text { Cu } & \text { Zn } \\ \text { Cs } & 495 & \mathbf{8 1 0} & 882 & \mathbf{7 3 8} \\ \text { K } & 209 & \mathbf{3 9 1} & \mathbf{3 5 0} & \mathbf{3 9 3}\end{array}$

Hierdurch erhebt sich ein berechtigter Zweifel, ob wir überhaupt ein Recht haben, schon aus der Reihenfolge der Doppelsulfate allein den Schlufs zu ziehen, dals die Löslichkeiten von den Atomgewichten unabbängig sind; denn in der $\mathrm{SO}_{4}$-Gruppe bescbränken wir uns auf ein Glied einer veränderlichen Gruppe; alle diese Salze werden auch von der $\mathrm{SeO}_{4}$-Gruppe gebildet. Die $\mathrm{Be}$ stimmung der Löslichkeiten der Doppelselenate kann eine durchaus andere Reihe geben, und die Kombination dieser letzteren mit der Reihe der Doppelsulfate kann für jedes Metall eine Konstante ergeben, die mit dem Atomgewicht in Übereinstimmung ist. Für die genaue Bestimmung dieser Konstanten jedoch sind drei oder vier Werte für jede Variable erforderlich. Diese Bedingung ist in der Reihe $\mathrm{M}_{2}{ }^{\mathrm{I}} \mathbf{M}^{\mathrm{II}}\left(\mathrm{RO}_{4}\right)_{2}$ nicht $\mathrm{zu}$ erfüllen, denn für $\mathrm{R}$ existiert nur Schwefel oder Selen. Zwei Salzreihen, die die geforderten Bedingungen erfüllen, sind: $\mathrm{M}_{2}{ }^{1} \mathrm{M}^{\mathrm{Iv}} \mathrm{X}_{6}$ und $\mathrm{M}^{\mathrm{II}} \mathrm{M}^{\mathrm{Iv}} \mathrm{X}_{6} \cdot 6 \mathrm{H}_{2} \mathrm{O}$, in denen $M^{\mathrm{I}}$ und $\mathrm{M}^{\mathrm{II}}$, sowie $\mathrm{M}^{\mathrm{IV}}$ und $\mathrm{X}$ durch verschiedene Elemente ersetzt 
werden können. Die Untersuchung dieser zwei Reihen möchte ich mir reservieren.

Ein zweiter Punkt, auf den die obigen Resultate hinweisen, betrifft die oft bemerkte, aber noch unerklärte Ähnlichkeit zwischen verschiedenen komplexen Radikalen und Elementen. Die Salze des Ammoniumradikals folgen in jeder Beziehung denselben Gesetzen wie die Alkalimetalle; wenn wir daher ein Klassifikationsschema ausarbeiten wollen, das auf Thatsachen und nicht auf vorgefafsten Ideen und Vorurteilen basiert ist, so können wir es für sicher annehmen, dafs - was auch immer die letzte Ursache für die Eigenschaften der Alkalisalze sein mag - genau die gleiche Ursache für die Ammonsalze malsgebend ist. Ammonium aber hat kein Atomgewicht und findet im System keinen Platz. Daraus folgt dann, dafs als letzte Basis für die Klassifikation nicht das Atomgewicht dienen kann, wie dies heute im periodischen System der Fall ist. Dafs eine lose Beziehung zwischen Atomgewicht und Eigenschaften existiert, ist ziemlich sicher; aber solange wir lediglich das periodische System als Mittel zur Klassifizierung benutzen, können Fortschritte nicht gemacht werden.

Sheffield, Chemical Laboratory, New-Haven, May 1902.

Bei der Redaktion eingegangen am 2. August 1902. 